Pesq. Vet. Bras. 37(10):1146-1152, outubro 2017

DOI: $10.1590 / \mathrm{S} 0100-736 \mathrm{X} 2017001000017$

\title{
Concentração de elementos traços em serpentes do litoral e da região serrana do Espírito Santo ${ }^{1}$
}

\author{
Alexandra Frossard ${ }^{2 *}$, Maria Tereza Weitzel Dias Carneiro ${ }^{3}$, Eduardo Lázaro de \\ Faria da Silva ${ }^{4}$, Claudio Barberine Camargo Filho ${ }^{5}$ e João Luiz Rossi Júnior ${ }^{6}$
}

\begin{abstract}
Frossard A., Carneiro M.T.W.D., Silva E.L.F., Camargo Filho C.B. \& Rossi Jr J.L. 2017. [Trace element concentration in snakes of coastal and serrana region of the State of Espírito Santo, Brazil.] Concentração de elementos traços em serpentes do litoral e da região serrana do Espírito Santo. Pesquisa Veterinária Brasileira 37(10):11461152. Programa de Pós-Graduação em Ecologia de Ecossistemas, Universidade Vila Velha, Rua Comissário José Dantas de Melo 21, Boa Vista, Vila Velha, ES 29102-920, Brazil. E-mail: xandfrossard@yahoo.com.br

Chemical elements are mostly involved in biochemical and geochemical cycles in concentrations which are harmless for organisms. However, accelerated human actions promote environmental changes such as the increase in contaminant intake. With this information, this study aimed to characterize the presence of trace elements in two snake species common in Southeastern Brazil, Boa constrictor and Bothrops jararaca. Copies of $B$. constrictor $(\mathrm{n}=18)$ were from pedestrian accidents which occurred on Highway stretch ES060 from Km 0 to Km 67.5. Copies of B. jararaca $(n=18)$ were captured by farmers in a rural mountainous area of Espíirito Santo State, Brazilo. They were analyzed 1 kidney fragment grass, autopsied specimens. Harvested kidneys were digested with acid mixture (HNO3 e $\mathrm{HCl} 1: 1)$ at $300^{\circ} \mathrm{C}, 40 \mathrm{~min}$ and quantification of microelementos (Cd, $\mathrm{Co}, \mathrm{Cr}, \mathrm{Cu}, \mathrm{Fe}, \mathrm{Mn}, \mathrm{Ni}$, $\mathrm{Pb}$ and $\mathrm{Zn}$ ) were determined by spectrometric optical emission with inductively coupled plasma (ICP OES). significant differences between males and females were observed, except for Chrome in B. constrictor ( $\mathrm{p}=0.03$ ), with an average of $1.6595 \mathrm{in} \mathrm{Cr}$ concentration of females and 0.2896 in males. However, when different species of snakes were compared, different concentrations were found for all elements, especially iron with a concentration of $106.2 \mathrm{mg} / \mathrm{g}$ in $B$. constrictor and $120.3 \mathrm{mg} / \mathrm{g}$ in B. jararaca; probably these animals came from areas near iron ore ports. The concentration of zinc in B. constrictor was $1261.8 \mathrm{mg} / \mathrm{g}$ and in B. jararaca $28,4 \mathrm{mg} / \mathrm{g}$. This study indicates that the analyzed snakes, which inhabit the regions of Greater Victoria and the mountainous region of the State of Espírito Santo have high concentrations of the microelements $\mathrm{Zn}$ and Fe.
\end{abstract}

INDEX TERMS: Bioacumulation, Boa constrictor, Bothrops jararaca, environmental contamination.

RESUMO.- Os elementos químicos, em sua maioria, encontram-se em ciclos bioquímicos e geoquímicos fechados e em concentrações que não causam efeitos nocivos aos or-

\footnotetext{
${ }^{1}$ Recebido em 13 de julho de 2016.

Aceito para publicação em 19 de janeiro de 2017.

${ }^{2}$ Programa de Pós-Graduação em Ecologia de Ecossistemas. Laboratório de Ictiologia Aplicada. Universidade Vila Velha (UVV). Rua Comissário José Dantas de Melo 21, Boa Vista, Vila Velha, ES 29102-920, Brasil. *Autor para correspondência: xandfrossard@yahoo.com.br

${ }^{3}$ Laboratório de Espectometria Aplicada. Universidade Federal do Espírito Santo (UFES), Avenida Fernando Ferrari. Vitória, ES, Brasil.
}

ganismos. Contudo, ações antrópicas aceleradas promovem alterações ambientais, como o aumento no aporte de contaminantes. Com essas informações, o presente trabalho

\footnotetext{
${ }^{4}$ Médico Veterinário.

${ }^{5}$ Programa de Pós-Graduação em Ecologia de Ecossistemas, Universidade Vila Velha (UVV) Rua Comissário José Dantas de Melo 21, Boa Vista, Vila Velha, ES 29102-920, Brasil.

${ }^{6}$ Programa de Pós-Graduação em Ecologia de Ecossistemas, Programa de Pós-Graduação em Ciência Animal, Hospital Veterinário Professor Dr. Ricardo Alexandre Hippler, Universidade Vila Velha (UVV) Rua Comissário José Dantas de Melo 21, Boa Vista, Vila Velha, ES 29102-920, Brasil.
} 
buscou caracterizar a presença aos elementos traços em duas espécies de serpentes comuns na região sudeste, Boa constrictor e Bothrops jararaca. Os exemplares de B. constrictor $(\mathrm{n}=18)$ foram provenientes de atropelamentos ocorridos no trecho da Rodovia ES-060 do $\mathrm{Km} 0$ ao $\mathrm{Km}$ 67,5. Os exemplares de $B$. jararaca $(\mathrm{n}=18)$ foram capturados por fazendeiros na zona rural da região serrana do Espírito Santo. Foram analisados 1 grama de fragmento de rim, de espécimes necropsiados. Os rins coletados foram digeridos com mistura ácida ( $\mathrm{HNO} ; \mathrm{HCl} ; 1: 1)$ a $300^{\circ} \mathrm{C}, 40 \mathrm{~min}$ e a quantificação de microelementos ( $\mathrm{Cd}, \mathrm{Co}, \mathrm{Cr}, \mathrm{Cu}, \mathrm{Fe}, \mathrm{Mn}$, $\mathrm{Ni}, \mathrm{Pb}$ e $\mathrm{Zn}$ ) foi realizada por espectrometria de emissão óptica com plasma indutivamente acoplado (ICP OES). Não foram observadas diferenças significativas entre machos e fêmeas, exceto para Cromo nas B. constrictor $(p=0,03)$, com média de 1,6595 nas concentrações de Cr nas fêmeas e 0,2896 em machos. Contudo, quando foram comparadas as diferentes espécies de serpentes, diferentes concentrações foram encontradas, para todos os elementos determinados, com destaque para o Ferro com uma concentração de $106,2 \mathrm{mg} / \mathrm{g}$ em B. constrictor e 120,3mg/g em B. jararaca, provavelmente por esses animais virem de áreas próximas à portos de minério de ferro. A concentração de Zinco em B. constrictor foi de $1261,8 \mathrm{mg} / \mathrm{g}$ e em B. jararaca foi de $28,4 \mathrm{mg} / \mathrm{g}$. 0 presente estudo indica que as serpentes analisadas, que habitam as regiões da Grande Vitória e serrana do Espírito Santo apresentam elevadas concentrações dos microelementos $\mathrm{Zn}$ e Fe.

TERMOS DE INDEXAÇÃO: Bioacumulação, Boa constrictor, Bothrops jararaca, contaminação ambiental.

\section{INTRODUÇÃO}

A degradação ambiental provocada pelo avanço das fronteiras agrícolas, atividade industrial e extração de petróleo afetam negativamente indivíduos e ecossistemas inteiros em todo o mundo, mesmo em áreas relativamente livres da intervenção antrópica, devido à dispersão global pela água e ar (Iwata et al. 1993). Inúmeros efeitos deletérios têm sido causados ao meio ambiente devido à urbanização acelerada e sem planejamento adequado. A alteração ou perda de habitat natural ou mesmo a redução da qualidade do ambiente é uma das consequências do crescimento urbano, e apresenta importantes implicações ecológicas, como os reflexos diretos na disponibilidade e qualidade do alimento, uma vez que as espécies diferem em sua capacidade de se adaptarem ao desenvolvimento humano.

0 avanço da fronteira agropecuária, extrativista e urbana gera uma perda gradual e contínua dos ambientes terrestres, afetando os recursos hídricos e nichos ecológicos a eles associados. Alguns organismos sofrem, primeiramente, com os efeitos que podem ser diretos, devido, por exemplo, à retirada das matas de galeria ou indiretos como a poluição e contaminação por meio do lixo industrial, doméstico, urbano ou de produtos agroquímicos e das águas de formação associadas à extração de petróleo.

Por serem usados demasiadamente nas atividades industriais, e por estarem presentes em defensivos agrícolas, os elementos traços podem ser responsáveis pela conta- minação do meio ambiente e estão disponíveis para biomagnificação através do ar e da água (Lagadic et al. 1998). Como resultado da contaminação, as serpentes podem acumular níveis elevados destes microelemetos, e de acordo com Burger et al. (2007), uma vez disponíveis no organismo desses répteis, estes elementos podem ser acumulados, principalmente em tecidos biológicos como músculos, rim, fígado e ponta de cauda.

Os organismos que vivem em zonas urbanas, industrializadas e rurais possuem risco potencial de serem expostos a elementos traços (Loumbourdis 1997). Como uma resposta a esse risco, os estudos com elementos traço têm se concentrado na ponderação dos efeitos nos organismos e nos ecossistemas (Oliveira et al. 1999). Uma forma muito comum de contaminação em áreas com influências antrópicas é por meio de elementos traços liberados por veículos automotores. 0 tráfego destes veículos libera metais através de desgaste de pneus e freios, vazamento de óleos e emissões gasosas (Sörme et al. 2002, Lough et al. 2005). A abundância desses contaminantes se relaciona ao volume do tráfego, à idade da frota e ao tipo de combustível empregado (Wanielista \& Youself 1993).

As proteções de aço nas rodovias, as placas de sinalização (galvanizadas, recobertas com Zinco) e as peças e baterias de automóveis também são fontes de elementos traços. Escapamentos de automóveis emitem poluentes, como Óxidos de Nitrogênio, Dióxido de Enxofre, Hidrocarbonetos, Dióxido e Monóxido de Carbono, aldeídos e material particulado (fuligem, poeira, fumaça e todo material suspenso no ar). Derramamentos de produtos químicos de caminhões e automóveis também são fontes de poluição química de estradas. Esses contaminantes em concentrações elevadas podem causar problemas fisiológicos e acumulativos nos animais e plantas (Spellerberg 1998).

Outra forma de contaminação é a aplicação constante de agroquímicos sobre o solo (Ramalho et al. 2000). Esta é uma prática comum nas áreas rurais para a proteção de lavouras para o controle de pragas, prática esta que causa a degradação química do solo e o acúmulo de elementos traços, principalmente o Manganês, Arsênio, Cobre, Zinco, Cobalto e Chumbo (Gimeno-Garcia et al. 1996), componentes ativos de vários agroquímicos (Tiller 1989). Já os fertilizantes minerais e orgânicos, bem como os corretivos do solo, contêm Zn, frequentemente como impurezas (Kiekens 1990) que também atuam nas concentrações desses elementos no solo (Ramalho et al. 2000), além de colaborar para a transferência desses poluentes na cadeia alimentar, assim como outros meios alternativos de convivência com os problemas gerados pelos excessos ocorridos (Oliveira et al. 1999).

As intoxicações por elementos traços mais frequentemente relatadas em serpentes estão associadas ao Selênio. Entretanto, outros metais que intoxicam os répteis são o Arsênio, Chumbo, Cádmio, Cromo, Mercúrio e Ferro (Hopkins et al. 2001, Burger et al. 2007). A detecção de elementos traços tem sido particularmente bem documentada para as cadeias alimentares aquáticas (Silva et al. 2015, Chovanec et al. 2003), mas apesar de serem reconhecidas como espécies bioindicadoras, os répteis ainda são caren- 
tes de estudos relacionados a teores de elementos traços (Hopkins et al. 2001, Burger et al. 2007), sejam animais de vida livre quanto cativos.

Deve-se considerar que a captura de animais de vida livre, como serpentes, envolve diversos fatores de riscos e dificuldades. Portanto, empregar métodos menos invasivos, como por exemplo, a utilização de animais provenientes de atropelamentos ou capturados e mortos por proprietários rurais e então encaminhados pelos órgãos ambientais, pode fornecer informações importantes sobre a ecologia das espécies, como a frequência de exposição aos contaminantes e a bioacumulação destes (Campbell et al. 2005).

Diante de todo o exposto, o presente trabalho visa caracterizar a exposição aos elementos traço (Cádmio-Cd, Cobalto-Co, Cromo-Cr, Cobre-Cu, Ferro-Fe, Manganês-Mn, Níquel-Ni, Chumpo-Pb e Zinco-Zn) de duas espécies de serpentes da Grande Vitória e da Região Serrana/ES, analisando frações de rim de espécimes necropsiados, partindo da hipótese de que as mesmas refletem os níveis de contaminação dos locais estudados, podendo então ser considerada um bioindicador ambiental.

\section{MATERIAL E MÉTODOS}

Serpentes da espécie Boa constrictor (jiboia) estudadas ( $\mathrm{n}=18)$, foram provenientes de atropelamentos ocorridos no trecho da Rodovia ES-060 que vai do Km 0, da Ponte Castelo Mendonça, em Vitória, até o Km 67,5, em Meaípe, conhecido como Rodovia do Sol no estado do Espírito Santo, e entregues ao Laboratório de Anatomia Animal, da Universidade Vila Velha (UVV-ES), nos anos de 2013 a 2014. (A licença do IBAMA no 19/06, do processo no 02009.000499/01-82) e o do IEMA no 67699286/20014).

Serpentes da espécie Bothrops jararaca (jararaca) estudas ( $n=18)$, foram encaminhadas pela comunidade rural do entorno do Criadouro Comercial de Serpentes Nickel, e posteriormente encaminhadas para pesquisa, por intermédio do IBAMA/ES. O Criador encontra-se em Melgaço, Distrito de Domingos Martins, Estado do Espírito Santo, Brasil. (Número de autorização 002/14-NUFAU/ DITEC/IBAMA/ES). Todas as serpentes vivas foram ambientadas e alojadas no Setor de Animais Silvestres do Hospital Veterinário Professor Dr. Ricardo Alexandre Hippler da UVV.

Área de estudo. A Rodovia do Sol atravessa os municípios de Guarapari e Vila Velha. Esta região compreende um clima tropical chuvoso com temperatura média de $18^{\circ} \mathrm{C}$, sendo fevereiro e julho os meses mais quente e frio, respectivamente. A vegetação marginal da rodovia é composta, principalmente por Restinga, a qual é considerada uma área fundamental para a biodiversidade, em espécies vegetais e animais presentes em grande parte da área adjacente à rodovia (INCAPER 2015). Ao longo do Sistema Rodovia do Sol existem três importantes reservas ambientais: Parque Natural Municipal de Jacarenema, Área de Preservação Ambiental de Setiba e Parque Estadual Paulo César Vinha (IPEMA 2004). Por estarem numa área de grande pressão antrópica, é necessário que exista um trabalho efetivo de educação ambiental nessas reservas.

O Município de Domingos Martins encontra-se na região central e serrana do Espírito Santo, com relevo acidentado, montanhoso, a cerca de $540 \mathrm{~m}$ de altitude. 0 clima é tropical de altitude com temperatura média anual inferior a $20^{\circ} \mathrm{C}$ devido à altitude do seu relevo ( $90 \%$ acima de $500 \mathrm{~m}$ ). Está localizado à latitude Sul de $20^{\circ} 21^{\prime} 44^{\prime \prime}$ e longitude Oeste de Greenwich de $40^{\circ} 39^{\prime} 36^{\prime \prime}$ e possui uma área de $1.228,353 \mathrm{Km}^{2}$, distando $43 \mathrm{~km}$ de Vitória. A vegetação predominante na região é a floresta ombrófila densa antimontana, com apenas uma unidade de conservação, o Parque
Estadual de Pedra Azul com uma área de 1.240 hectares, localizada no distrito de Aracê. Porém, por Domingos Martins ser uma área de relevante interesse ecológico, todo o município faz parte do Corredor Central da Mata Atlântica, e por ser uma área prioritária de conservação da Mata Atlântica no Espírito Santo faz parte do Corredor Ecológico Pedra Azul x Forno Grande (PMPEPA 2004). Seu atual desenvolvimento se deve ao turismo e à agricultura, com o café e hortifrutigranjeiros (IBGE 2014, PMSDM 2013). Muitos proprietários e trabalhadores rurais que se situam nas proximidades, são responsáveis por recolher e enviar serpentes para o criador comercial de Melgaço, distrito do município.

As serpentes. As Boa constrictor, da família Boidae, possuem ampla distribuição na América do Sul, e habitam áreas florestadas e também urbanas (Carvalho \& Nogueira 1998). Podem ser terrestres e semi-arborícolas e geralmente são ativas à noite, possuem receptores térmicos labiais, sensíveis a raios infravermelhos emitidos pela presa em potencial, e quando capturadas, são mortas por constrição. As serpentes possuem adaptações cranianas que permitem a ingestão de presas relativamente grandes, e itens alimentares consumidos são geralmente baseados em aves e mamíferos, podendo se alimentar também de lagartos e anfíbios (Scartozzoni \& Molina 2004). Como foram obtidos,10 exemplares de fêmeas de Boa constrictor e 8 exemplares de machos, analisamos a bioacumulação dos elementos traços nos rins entre o sexo dessa espécie.

As Bothrops jararaca, da família Viperidae, tem distribuição ampla e associada ao domínio da Mata Atlântica, estendendo-se por ambiente florestais e áreas antropizadas. Com hábitos principais, terrestres e noturnos, se alimentam basicamente de roedores (Hartmann et al. 2003). Possui em sua dentição aparelho inoculador do tipo solenóglifo e se caracteriza por possuir cabeça triangular recoberta por escamas pequenas e presença de fosseta loreal. As serpentes do gênero Bothrops são caracterizadas por possuírem a cauda sem maiores modificações, geralmente com escamas subcaudais em pares (Cardoso et al. 2009).

As Bothrops jararacas adquiridas Criadouro Comercial de Serpentes Nickel, foram acomodadas em "serpentários" improvisados para realização de manejo intensivo, criado pra receber um variado número de espécies de animais silvestres, provenientes de diversos climas, em uma área bastante reduzida. Proporcionamos uma forma simples de manutenção, com facilidade para a vigilância dos animais, em relação à alimentação e para o controle dos fatores ambientais como temperatura e umidade. Esse sistema, porém, com espaço reduzido, apresentou desvantagens, como a impossibilidade das serpentes realizarem termorregulação apropriada, obrigando a uma climatização em parâmetros não adequados para todos os espécimes. A falta de contato com elementos naturais não permitiu um perfeito equilíbrio fisiológico das serpentes. Isso se tornou evidente pela facilidade com que os animais adoeceram, e morreram, talvez também o surgimento de enfermidades contagiosas que, não diagnosticamos e, portanto, não as controlamos. Além de tudo, os animais não passaram por quarentena, como em outros serpentários. Além desses, vários foram os motivos para as mortes da B. jararaca, como lesões traumáticas, às vezes de bastante gravidade, produzidas freqüentemente no ato da captura, e além desses, as serpentes apresentam grande variedade de doenças, sendo comum as infecciosas e as parasitárias, que se agravaram pelo estresse e pelos longos períodos de permanência em condições artificiais além de estarem sendo submetidos a manipulação (Melgarejo-Giménez 2002). Portanto, após mortas, foram utilizadas para o presente estudo.

Preparação das amostras. Foram utilizadas 18 serpentes da espécie Boa constrictor, e 18 serpentes da espécie Bothrops jarara$c a$. Todos os espécimes mortos foram imediatamente congelados até a realização da necropsia, a fim de se coletar frações do rim. 
As 18 jiboias (Boa constrictor) encontradas atropeladas e mortas foram encaminhadas já congeladas ao Laboratório de Anatomia Animal da UVV e mantidas a $-20^{\circ} \mathrm{C}$ até a necropsia. As 18 jararacas (Bothrops jararaca) utilizadas, doadas pelo criador, foram trazidas vivas até o Setor de Animais Silvestres do Hospital Veterinário da UVV e lá manejadas de acordo com o as normas de uso e manejo da fauna silvestre em cativeiro em território brasileiro (IBAMA 2008). Mas alguns indivíduos foram a óbito por má adaptação ao cativeiro e, portanto, utilizadas para a esse estudo de análise de elementos traços.

Após a realização da necropsia, os fragmentos coletados de rim, com aproximadamente 1 grama de tecido, foram armazenados individualmente em tubo de microtitulação tipo Eppendorf® a $-80^{\circ} \mathrm{C}$ devidamente identificados. Para a digestão de todos os tecidos, foram preparadas soluções contendo $5 \mathrm{ml}$ de ácido nítrico suprapuro $5 \mathrm{M}$ (Merck) e $5 \mathrm{ml}$ de ácido clorídrico $5 \mathrm{M}$ (Merck) (Perez-Lopez 2008 - adaptado).

Esses processos foram preparados em tubos digestores previamente lavados com solução de ácido nítrico a $10 \%$ e levados ao aparelho digestor (Marconi Mod. MA 851), por aproximadamente 4 horas a uma temperatura máxima de $180^{\circ} \mathrm{C}$ (Burger et al. 2006 adaptado), até que a solução estivesse límpida e translucida. Esta solução resultante foi transferida para tubos Falcon de $15 \mathrm{ml}$ após serem filtradas, e então teve o volume final completado com água ultra-pura, tipo 1 (água Mili-Q).

A quantificação dos elementos traço (Cd, Co, Cr, Cu, Fe, Mn, Ni, $\mathrm{Pb}$ e $\mathrm{Zn}$ ) foi realizada em um padrão multielementar por espectrômetro de emissão óptica com plasma indutivamente acoplado (ICP-OES), modelo Optima 7000 da Perkin Elmer, com coeficiente de variação sempre menor que 10\%, margem que é aceitável (Wylie et al. 2009 - adaptado). Os limites de detecção (LD) e quan-

Quadro 1. Limites de detecção (LD) e Limites de quantificação (LQ) de cada elemento traço analisado pelo ICO-OES $(\mu \mathrm{g} / \mathrm{L})$

\begin{tabular}{ccc}
\hline $\begin{array}{c}\text { Elementos } \\
\text { traços }\end{array}$ & $\begin{array}{c}\text { Limites de } \\
\text { detecção }\end{array}$ & $\begin{array}{c}\text { Limite de } \\
\text { quantificação }\end{array}$ \\
\hline $\mathrm{Cd}$ & 0,1579 & 0,52107 \\
$\mathrm{Co}$ & 0,2359 & 0,77847 \\
$\mathrm{Cr}$ & 0,1208 & 0,39864 \\
$\mathrm{Cu}$ & 0,1602 & 0,52866 \\
$\mathrm{Fe}$ & 0,0581 & 0,19173 \\
$\mathrm{Mn}$ & 0,0257 & 0,08481 \\
$\mathrm{Ni}$ & 0,2338 & 0,77154 \\
$\mathrm{~Pb}$ & 1,118 & 3,6894 \\
$\mathrm{Zn}$ & 0,2263 & 0,74679
\end{tabular}

tificação (LQ) para os metais analisados foram expressos no Quadro 1.

Análises estatísticas. Foram realizados Testes de t-Student e de Mann-Whitney para avaliar as concentrações de elementos traço nos rins entre machos e fêmeas de $B$. constrictor com nível de significância de $p<0,05$, e para observar as concentrações de elementos traços nos rins das duas diferentes espécies da região de montanha e do litoral, a mediana e as concentrações máximas e mínimas foram expressas em tabela.

\section{RESULTADOS E DISCUSSÃO}

Foram obtidas um total de 36 amostras, divididas em duas famílias de espécies diferentes de serpentes, 18 amostras de rim da Boidae: Boa constrictor e 18 amostras de rim da Viperidae: Bothrops jararaca.

No Quadro 2 expressam-se os valores médios e medianos das concentrações dos elementos traços presentes nos rins das serpentes estudadas. As medianas das concentrações nos representantes de $B$. jararaca foram as mais altas para seis dos elementos traços analisados, sendo somente três medianas são maiores para as $B$. constrictor encontradas na região litorânea. Concentrações elevadas de $\mathrm{Cd}$ e $\mathrm{Pb}$, que são metais não essenciais, foram encontradas nas duas espécies analisadas, com destaque para a maior concentração de $\mathrm{Pb}$. Os elementos traço não se diferem significativamente entre machos e fêmeas de jiboias, exceto para o Cromo, que é um metal essencial $\left(\mathrm{p}=0,031^{*}\right)$. Com média de 1,6595 nas concentrações de Cr nas fêmeas e 0,2896 em machos (Quadro 2).

Os elementos traços encontraram-se sem diferenças para machos e fêmeas de grande parte dos elementos quantificados nos rins de B. constrictor analisados. Embora, o número de fêmea seja maior do que jiboias macho, as concentrações de elementos traços não se diferenciaram entre os sexos exceto para o Cr (Quadro 2). Ohlendorf et al. (1988) também não encontraram uma correlação significativa entre as concentrações das serpentes Gopher (Pituophis melanoleucus). Campbell (2005) encontrou diferenças significativas para o selênio e mercúrio, elementos não analisados no presente estudo, em serpente d'água peçonhenta, onde machos, possuíam níveis significativamente maiores do que fêmeas, no fígado e rins. Diferenças específicas de cada sexo, na acumulação de elementos traços

Quadro 2. Mediana, mínimo e máximo valores de teor de elementos traços nos rins de Boa constrictor encontradas atropeladas na Rodovia do sol e Bothrops jararaca encontradas em áreas de produção agrícola no município de Domingos Martins, ES

\begin{tabular}{|c|c|c|c|c|c|c|}
\hline \multicolumn{4}{|c|}{ B. constrictor do litoral } & \multicolumn{3}{|c|}{ B. jararaca de montanha } \\
\hline & Mediana & Mín - Máx & $q$ e $\widehat{\jmath}(\mathrm{P}>0,05)$ & & Mediana & Mín - Máx \\
\hline $\mathrm{Cd}$ & 0,0360 & $0-0,095$ & $P=0,058$ & $\mathrm{Cd}$ & 0,1813 & $0,015-1,05$ \\
\hline Co & 0,0451 & $0,015-0,231$ & $P=0,328$ & Co & 0,0815 & $0,015-0,397$ \\
\hline $\mathrm{Cr}$ & 0,9131 & $0,0527-12,421$ & $P=0,031^{*}$ & $\mathrm{Cr}$ & 1,6967 & $0,12-8,451$ \\
\hline $\mathrm{Cu}$ & 3,1823 & $1,335-9,264$ & $P=0,450$ & $\mathrm{Cu}$ & 2,9018 & $0,87-16,492$ \\
\hline $\mathrm{Fe}$ & 106,1860 & $26,685-275,7$ & $P=0,505$ & $\mathrm{Fe}$ & 120,3421 & $30,96373,535$ \\
\hline $\mathrm{Mn}$ & 0,7739 & $0,225-1,83$ & $P=0,725$ & $\mathrm{Mn}$ & 0,6323 & $0,165-1,963$ \\
\hline $\mathrm{Ni}$ & 0,6923 & $0,015-6,616$ & $P=0,505$ & $\mathrm{Ni}$ & 0,9155 & $0,039-8,057$ \\
\hline $\mathrm{Pb}$ & 1,2081 & $0,165-7,894$ & $P=0,450$ & $\mathrm{~Pb}$ & 1,5023 & $0,225-5,240$ \\
\hline $\mathrm{Zn}$ & 1261,8396 & $12,773-28418,28$ & $P=0,083$ & $\mathrm{Zn}$ & 28,3597 & $10,395-74,778$ \\
\hline
\end{tabular}

Todos os valores estão expressos em $\mathrm{mg} / \mathrm{g}$. 
ocorreu em um estudo controlado de laboratório com juvenis de cobras d'água (Hopkins et al. 2002). As diferenças na acumulação de metais em serpentes fêmeas e machos pode ocorrer por causa de diferenças em função do sexo na preferência alimentar, tamanho do corpo, ou a eliminação durante a reprodução; por exemplo, as serpentes fêmeas transferem os metais para seus ovos (Burger 1992, Hopkins et al. 2002, 2004).

O Cd é um elemento traço não essencial e pode causar graves efeitos negativos à saúde dos animais, embora répteis e mamíferos apresentem maior resistência aos seus efeitos tóxicos quando comparados a outros vertebrados (Eisler 1985). A origem do Cd no ambiente ocorre principalmente, por fontes antropogênicas e, bioacumula em maiores concentrações, nos rins e fígado. Teores representativos deste metal foram registrados no fígado, músculo e pele (Campbell et al. 2005). No presente estudo, não foram encontradas concentrações elevadas deste elemento no rim das serpentes analisadas, o que sugere que este único tecido não pode ser um biomarcador deste metal do organismo. Burger et al. (2005) afirmam que níveis sanguíneos de Cd podem ser indicadores de exposição recente, mas o metal pode se acumular em tecidos moles e, alguns estudos afirmam que os principais órgãos-alvo para a acumulação de Cd em mamíferos e outros vertebrados, incluindo répteis, são o fígado, rim e da mucosa intestinal (Gutleb \& Gutleb 1991, Cooke \& Johnson 1996, Linder \& Grillitsch 2000, Campbell et al. 2005, Mann et al. 2006).

Campbell et al. (2005) sugerem que as concentrações de Cd acima de $0,44 \mu \mathrm{g} / \mathrm{g}$ no rim podem indicar uma grande exposição ambiental a esse metal e, grande parte das espécies de serpentes estudas na presente pesquisa, apresentaram concentrações bem abaixo do que a sugerida pelo referido autor. Estes resultados corroboram com os resultados encontrados por Hopkins et al. (2001), quando comparados aos animais controle de seus experimentos com serpentes aquáticas. 0 que nos permite supor que os níveis desses elementos se encontram em baixas concentrações, tanto na região serrana, quanto no litoral.

$0 \mathrm{~Pb}$ é um elemento altamente tóxico que pode causar mortalidade de animais selvagens (Mateo et al. 1998). De acordo com Kalisinska et al. (2004), uma pequena quantidade de $\mathrm{Pb}$ no cérebro pode alterar o comportamento das aves ao ponto de colocar em perigo a sua sobrevivência e afetar o sucesso reprodutivo. Pain et al. (1995) estabeleceram faixas de concentrações de $\mathrm{Pb}$ em tecido hepático e renais, onde determinaram que concentrações abaixo de 2 $\mu \mathrm{g} / \mathrm{L}$ podem ser indicativos de uma baixa exposição ao metal. Já concentrações entre 6 e $20 \mu \mathrm{g} / \mathrm{L}$ estão associadas a alta exposição, podendo causar efeitos clínicos. Todos esses dados ainda não foram determinados para muitos dos répteis, incluindo as serpentes. No presente trabalho, foi possível observar baixas concentrações de $\mathrm{Pb}$ tanto nas jiboias quanto nas jararacas, indicando que as mesmas podem não estar sofrendo com a exposição a esse metal.

Burger et al. (2005) analisando tecidos de cobra d'água (Nerodia sipedon) não encontraram concentrações significativamente altas de $\mathrm{Pb}$ em tecido renal, o que difere do presente estudo. Campbell et al. (2005) encontraram con- centrações máximas de $\mathrm{Pb}$ em tecidos renais de $31 \mu \mathrm{g} / \mathrm{g}$ em um exemplar de Nerodia sipedon, resultado similar a um estudo realizado por Burger et al. (2006), que encontraram concentrações mais altas em Akistrodon piscivorous do que em Nerodia fasciata. Ambos estudos detectaram concentrações consideradas letais, o que não corrobora com o presente trabalho.

Para metais essenciais, como o Co, $\mathrm{Cr}, \mathrm{Cu}, \mathrm{Fe}, \mathrm{Mn}, \mathrm{Ni}$, e $\mathrm{Zn}$, não se encontrou valores limites (Taggart et al. 2006), principalmente para os répteis (Hopkins 2000). Esses metais participam de alguns processos fisiológicos nos organismos (Carapeto 2012). O cobalto é um elemento químico essencial, presente na vitamina B12, com utilização principal na indústria metalúrgica para produção de aços com características especiais de dureza e resistência, como catalisador na indústria química e de óleos e na forma de sais, na indústria de cerâmica, como pigmentos. Porém, quando em excesso, os efeitos tóxicos observados nas exposições a diferentes compostos de cobalto são mais pronunciados nos pulmões humanos, na forma de asma brônquica e fibrose (Alves \& Rosa 2003). No presente estudo, tanto nas jiboias quanto nas jararacas as médianas das concentrações de Co encontradas foram maiores do que as relatadas por Burger et al. (2006). Já para o Cr, as médias das concentrações encontradas foram menores do que as relatadas por Burger et al. (2006), Burger et al. (2005), Campbell et al. (2005) e Hopkins (2001).

O cobre em concentrações elevadas é prejudicial à saúde, porém, em pequenas quantidades é benéfico ao organismo humano, catalisando a assimilação do ferro e seu aproveitamento na síntese da hemoglobina do sangue, facilitando a cura de anemias (Eisler 2000). As concentrações de cobre encontradas no presente estudo estão relativamente baixas, para as duas espécies de serpentes estudadas e são menores do que as encontradas por Hopkins et al. (2001), quando avaliaram tecidos da cobra d'água (Nerodia fasciata). 0 cobre no meio ambiente é proveniente de corrosão de efluentes de estações de tratamento de esgotos, uso de compostos de cobre como algicidas aquáticos, escoamento superficial (Pacheco 2009).

As médias das concentrações de Fe, principalmente nas jiboias, provavelmente por virem de áreas próximas à portos de minério de ferro, foram consideravelmente menores do que as encontradas por Burger et al. (2006) quando analisaram tecidos de serpentes aquáticas não peçonhentas (Nerodia fasciata e $N$. taxispilota) e peçonhentas (Akistrodon piscivorous) que encontraram 663 e 1779 ( $\mu \mathrm{g} / \mathrm{g})$. Já para o elemento traço $\mathrm{Mn}$ e $\mathrm{Ni}$, as médias das concentrações encontradas foram consideravelmente maiores do que as relatadas por Burger et al. (2006).

As médias das concentrações de Zn presentes em tecido renal das serpentes do presente estudo foram muito altos, semelhantes às encontradas por Perez-Lopes et al. (2008). Levengood et al. (1999) encontraram uma grande faixa de variação nas concentrações de $\mathrm{Zn}$ no fígado e no rim (473- $1990 \mu \mathrm{g} / \mathrm{g}$ ) que causaram efeitos tóxicos em patos selvagens. Para aves marinhas, Perez-Lopes et al. (2008) sugerem que concentrações hepáticas acima de $200 \mu \mathrm{g} / \mathrm{g}$ podem ser tóxicas. No presente estudo, as concentrações 
de Zn nas jibóias da região litorânea foi consideravelmente maior do que nas jararacas encontradas na região serrana, o que nos permite entender que esse elemento se encontra em concentrações muito altas nessa região.

Os efeitos tóxicos de $\mathrm{Zn}$ atuam sobre o sistema respiratório dos animais marinhos e nos representantes do topo de cadeia. É um elemento largamente utilizado na indústria, principalmente em galvanoplastias, na forma metálica e de sais, tais como cloreto, sulfato, cianeto, etc. Pode entrar no ambiente através de processos naturais (lixiviação de rochas e solos) e antropogênicos, entre os quais se destaca a produção de ferro e aço e os efluentes domésticos (Carvalho et al. 2008). Podemos supor então que os níveis renais de Zn podem estar causando problemas clínicos nas serpentes.

As principais fontes de $\mathrm{Pb}, \mathrm{Zn}$ e $\mathrm{Cu}$ no ambiente são, provavelmente, de origem industrial e relacionadas ao tráfego de veículos. Apesar da adição de Pb ser proibida em combustíveis em alguns países mais desenvolvidos, as emissões relacionadas a esse metal podem ser provenientes da abrasão de pneus em superfícies de estrada, desgaste das pastilhas de freio e dos pneus dos automóveis (Zechemeister et al. 2006, Carvajal et al. 2010). As proteções de aço nas rodovias, as placas de sinalização (galvanizadas, recobertas com zinco) e as peças e baterias de automóveis também são fontes de metais pesados (Sörme et al. 2002, Lough et al. 2005).

Devido à vulnerabilidade e a grande variedade de contaminantes ambientais, as serpentes têm sido usadas como indicadores ambientais, em menores escalas, em várias partes do mundo (Burger et al. 2006, Burger et al. 2005, Campbell et al. 2005, Hopkins 2001). Porém, no Brasil os estudos ainda são escassos, o que dificulta a comparação entre espécies residentes e os locais de contaminação. 0 presente estudo é de extrema importância, pois antes nunca havia sido avaliado grau de contaminação das espécies em questão tanto na região da Grande Vitória quanto na região serrana/ES.

\section{CONCLUSÕES}

0 presente estudo indica que as serpentes analisadas, que habitam as regiões da Grande Vitória e região serrana/ES apresentam elevadas concentrações de substâncias inorgânicas tóxicas, como o Zn.

As duas espécies de serpentes estudadas apresentaram concentrações de Zn consideradas toxicas, podendo ser uma das causas das mortes desses animais.

As concentrações de metais encontradas estão de acordo com outros estudos, mas por serem essenciais ao organismo, é difícil mensurar qual concentração poderia causar efeitos realmente tóxicos para os répteis.

Os resultados apresentados nos permitem afirmar que as espécies de serpentes podem ser utilizadas como indicadores da qualidade ambiental.

\section{REFERÊNCIAS}

Alves A.N.L. \& Rosa H.V.D. 2003. Exposição ocupacional ao cobalto: aspectos toxicológicos. Braz. J. Pharmaceut. Sciences 39:2-3.

Burger J. 1992. Trace element levels in pine snake hatchlings: tissue and temporal differences. Arch. Environ. Contam. Toxicol. 22:209-213.

Burger J., Campbell K.R., Murray R., Campbell T.S., Gaines K.F., Jeitner C.,
Shukla T., Burke S. \& Gochfeld M. 2007. Metal levels in blood, muscle and liver of water snakes (Nerodia spp.) from New Jersey, Tennessee and South Carolina. Sci. Total Environ. 373(2/3):556-563.

Burger J., Campbell K.R., Campbell T.S., Shukla T., Jeitner C. \& Gochfeld M. 2005. The use of skin and blood as non-destructive indicators of heavy metal contamination in northern water snakes (Nerodia sipedon). Arch. Environ. Contam. Toxicol. 49:232-238.

Burger J., Murray S., Gaines K.F., Novak J.M., Punshon T., Dixon C. \& Gochfeld M. 2006. Element levels in snakes in South Carolina: differences between a control site and exposed site on the Savannah River Site. Environ. Monitor. Assessment 112:35-52.

Campbell K.R., Campbell T.S. \& Burger J. 2005. Heavy metal concentrations in northern water snakes (Nerodia sipedon) from East Fork poplar creek and the Little River, East Tennessee, USA. Arch. Environ. Contam. Toxicol. 49:239-248.

Cardoso J.L.C., França F.O.S., Wen F.H., Málaque C.M.S. \& Haddad Jr V. 2009. Animais peçonhentos no Brasil: biologia, clínica e terapêutica dos acidentes. Vol.2. Sarvier, São Paulo, p.540.

Carapeto C. 2012. Metais pesados. Disponível em <http://www.antonio-fonseca.com/Unidades\%20Curriculares/2-Ano/Poluicao/1\%20Licoes/13\%20-\%2014\%20-\%2015/METAIS.pdf> Acesso em 15 dez. 2012.

Carvalho M.A. \& Nogueira F. 1998. Serpentes da área urbana de Cuiabá, Mato Grosso: aspectos ecológicos e acidentes ofídicos associados. Cad. Saúde Pública, Rio de J., 14(4):753-763.

Carvalho C.E.V., Di Beneditto A.P.M., Souza C.M.M., Ramos R.M.A. \& Rezende C.E. 2008. Heavy metal distribution in two cetacean species from Rio de Janeiro State, south-eastern Brazil. J. Marine Biol. Assoc. United Kingdom 88(6):1117-1120.

Cavajal B., Aboal J.R., Fernandez J.A., Real C. \& Carballeira A. 2010. Influence of roads and inhabited áreas on metal concentrations in terrestrial mosses. Atmospheric Environ. 44:3432-3441.

Chovanec A., Hofer R. \& Schiemer F. 2003. Fish as bioindicators and biomonitors. Trace Metals and other Contaminants in the Environment 6:639-676.

Cooke J.A. \& Johnson M.S. 1996. Cadmium in small mammals, p.377-388. In: Beyer W.N., Heinz G.H. \& Redmon-Norwood A.W. (Eds), Environmental Contaminants in Wildlife: interpreting tissue concentrations. Lewis Publishers, Boca Raton, FL.

Eisler R. 1985. Cadmium hazards to fish, wildlife, and invertebrates: a synoptic review. U.S. Fish and Wildlife Service Biological Report. Patuxent Wildlife Research Center, Laurel, Maryland. Contaminant Hazard Reviews Report 2. Biological Report 85(1.2).

Eisler R. 2000. Copper, p.93-200. In: Ibid. (Ed.), Handbook of Chemical Risk Assessment: health hazards to humans, plants, and animals. Vol.1. Metals. 738p. Lewis Publishers. Boca Raton.

Gimeno Garcia E., Abreu V. \& Boluda R. 1996. Heavy metals incidence in the aplications of inorganic fertilizers and pesticides to rice farming soil. Enviromental Pollution, Kidlington, 92(1):19-25.

Gutleb A.C. \& Gutleb B. 1991. Rückstande von Cadmium in Wirbeltieren aus dem Koflachtal/Kärnten. Carinthia II 181(101):609-616.

Hartmann P.A., Hartmann M.T. \& Giasson L.O.M. 2003. Uso do hábitat e alimentação em juvenis de Bothrops jararaca (Serpentes, Viperidae) na Mata Atlântica do sudeste do Brasil. Phyllomedusa 2(1):35-41.

Hopkins W.A. 2000. Reptile toxicology: challenges and opportunities on the last frontier in vertebrate ecotoxicology. Environ. Toxicol. Chem. 19:2391-2393.

Hopkins W.A., Roe J.H., Snodgrass J.W., Jackson B.P., Kling D.E., Rowe L.C. \& Congdon J.D. 2001. Non-destructive indices of trace element exposure in squamate reptile. Environ. Polution 115:1-7.

Hopkins W.A., Roe J.H., Snodgrass J.W., Staub B.P., Jackson B.P. \& Congdon J.D. 2002. Effects of chronic dietary exposure to trace elements on banded water snakes (Nerodia fasciata). Environ. Toxicol. Chem. 21:906-913.

Hopkins W.A., Staub B.P., Baionno J.A., Jackson B.P., Roe J.H. \& Ford N.B. 2004. Trophic and maternal transfer of selenium in brown house snakes (Lamprophis fuliginosus). Ecotoxicol. Environ. Safety 58:285-293.

IBGE 2014. Cidades. Instituto Brasileiro de Geografia e Estatística. Dis- 
ponível em <http://www.cidades.ibge.gov.br/painel/historico.php ?lang $=\& \operatorname{codmun}=320190 \&$ search $=$ espirito-santo $\mid$ domingos - mar tins|infograficos:-historico > Acesso em $27 \mathrm{dez} 2014>$

IBAMA 2008. Instrução Normativa 169. Disponível em <http://www.icmbio.gov.br/sisbio/images/stories/instrucoes_normativas/IN\%20n\%20 169\%20manejo\%20ex\%20situ.pdf> Acesso em 2 mar. 2015.

INCAPER 2015. Instituto Capixaba de Pesquisa, Assistência Técnica e Extensão Rural. Disponível em <http://hidrometeorologia.incaper.es.gov. br/caracterizacao/guarapari_carac.php> Acesso em 22 dez. 2015.

IPEMA 2004. Conservação da Mata Atlântica no Espírito Santo: cobertura florestal, unidades de conservação e fauna ameaçada (Programa: Centros para a Conservação da Biodiversidade Conservação Internacional do Brasil). II título. Instituto de Pesquisas da Mata Atlântica, Vitória. 112p.

Kalisińska E., Salicki W., Mysłek P., Kavetska K.M. \& Jackowski A. 2004. Using the Mallard to biomonitor heavy metal contamination of wetlands in north-western Poland. Science of The Total Environment. 320(23):145-161.

Kiekens L. 1990. Zinc, p.261-277. In: Alloway B.J. (Ed.), Heavy Metals in Soils. Blackie and Son, Glasgow.

Lagadic L., Caquet T., Amiard J.C. \& Ramade F. 1998. Utilisation de Biomarqueur Spourla Surveillance de la Qualite de l'Environnement. Lavoisier, Tec and Doc, Paris.

Levengood J.M., Sanderson G.C., Anderson W.L., Foley G.L., Skowron L.M., Brown P.W. \& Seets J.W. 1999. Acute toxicity of ingested zinc shot in game-farm mallards. Ill. Nat. Hist. Surv. Bull. 36:1-36.

Linder G. \& Grillitsch B. 2000. Ecotoxicology of metals, p.325-459. In: Sparling D.W., Linder G. \& Bishop C.A. (Eds), Ecotoxicology of Amphibians and Reptiles. Society of Environmental Toxicology and Chemistry (SETAC), Pensacola, FL.

Lough G.C., Chauer J.J., Park J.S., Shafer S.M., Deminter J. \& Weinstein J. 2005. Emissions of metals associated with motor vehicle roadway. Environ. Chem. Technol. 39:826-836.

Loumbourdis N.S. 1997. Heavy metal concentration in a lizard, Agama stelliostellio, compared in urban, high altitude and agricultural, low altitude areas of north Greece. Bull. Environ. Contamin. Toxicol. 58:945-952. <http://link.springer.com/article/10.1007/s001289900426\#page-1>

Mann R.M., Serra E.A. \& Soares A.M.V.M. 2006. Assimilation of cadmium in a European lacertid lizard: is trophic transfer important? Environ. Toxicol. Chem. 25:3199-3203.

Mateo R., Estrada J., Paquet J.Y., Riera X., Domínguez L., Guitart R. \& Martinez-Vilalta A. 1999. Lead shot ingestion by marsh harriers Circus aeruginosus from Ebro delta, Spain. Environ. Pollut. 104:435-440.

Melgarejo-Giménez A.R. 2002. Criação e manejo de serpentes, p.175-199. In: Andrade A., Pinto S.C. \& Oliveira R.S. (Eds), Animais de Laboratório: criação e experimentação [online]. Editora Fiocruz, Rio de Janeiro. 468p. ISBN: 85-7541-015-6. Available from SciELO Books.

Ohlendorf H.M., Hothem R.L. \& Aldrich T.W. 1988. Bioaccumulation of selenium by snakes and frogs in the San Joaquin Valley, California. Copeia 704-710. Published by American Society of Ichthyologists and Herpetologists (ASIH). DOI: 10.2307/1445391

Oliveira T.S., Costa L.M., Cruz C.D. \& Horn H.A. 1999. Metais pesados como indicadores de materiais de origem em uma topolitoseqüência do tri- ângulo mineiro, estado de minas gerais. Pesq. Agropec. Bras. 34:14511465.

Pacheco A. 2009. Avaliação das concentrações de metais pesados no sedimento, água e em Leucopternis lacernulata (gavião pomba), Baía de Sepetiba, Rio de Janeiro: estudo de caso. Gaia Scientia 3(2):23-31.

Pain D.J., Sears J. \& Newton I. 1995. Lead concentration in birds of prey in Britain. Environ. Pollut. 87:173-180.

Perez-Lopez M., Mendonza M.H., Beceiro A.L. \& Rodriguez F.S. 2008. Heavy metal (Cd, $\mathrm{Pb}, \mathrm{Zn})$ and metalloid (As) content in raptor species from Galicia (NW Spain). Ecotoxicol. Environ. Safety 70:154-162

PMSDM 2014. Plano Municipal de Saúde de Domingos Martins 20131015. Disponível em <http://www.domingosmartins.es.gov.br/files/ PDF/Plano_Municipal_Saude_2014_2017.pdf> Acesso em 27 dez. 2014.

Plano de Manejo do Parque Estadual da Pedra Azul 2004. Instituto de Defesa Agropecuária e Florestal do Espírito Santo, CEPEMAR.

Perez-Lopez M., Mendonza M.H., Beceiro A.L. \& Rodriguez F.S. 2008. Heavy metal $(\mathrm{Cd}, \mathrm{Pb}, \mathrm{Zn})$ and metalloid (As) content in raptor species from Galicia (NW Spain). Ecotoxicol. Environ. Safety 70:154-162.

Ramalho J.F., Garcia P., Amaral S. \& Nelson M.B.V. 2000. Ary C >X>. Contaminação da microbacia de Caetés com metais pesados pelo uso de agroquímicos. Pesq. Agropec. Bras. [online]. Vol.35, no.7, p.1289-1303. ISSN 1678-3921.

Scartozzoni R.R. \& Molina F.B. 2004. Comportamento alimentar de Boa constrictor, Epicrates cenchria e Corallus hortulanus (Serpentes: Boidae) em cativeiro. Revta Etologia 6(1):25-31. ISSN 1517-2805

Sílvia P., Duarte B., Castro N., Almeida P.R., Caçador I. \& Costa J.L. 2015. The Lusitanian toadfish as bioindicator of estuarine sediment metal burden: The influence of gender and reproductive metabolism. Ecological Indicators 48:370-379.

Sörme L. \& Lagerkvist R. 2002. Sources of heavy metals in urban wastewater in Stockholm. Sci. Total Environ. 298:131-145.

Spellerberg I.F. 1998. Ecological effects of roads and traffic: a literature review. Global Ecology and Biogeography Letters 7(3):17-333.

Taggart M.A., Figuerola J., Green A.J., Mateo R., Deacon C., Osborn D. \& Meharg A.A. 2006. After the Aznalco' llar mine spill: arsenic, zinc, selenium, lead and copper levels in the livers and bones of five waterfowl species. Environ. Res. 100:349-361.

Tiller K.G. 1989. Heavy metals in soils and their environmental significance, p.113-114. In: Tiller K.G. (Ed.), Advances in Soil Science. Vol.9. Springer, New York.

Wanielista P.M. \& Youse A.Y. 1993. Receiving water quality, p.159-211. In: Ibid. (Eds), Stormwater management. Publications John Wiley, New York.

Wylie G.G.D., Hothem R.L., Bergen D.R., Martin L.L., Taylor R.J. \& Brussee B.E. 2009. Metals and trace elements in Giant Garter snakes (Thamnophis gigas) from the Sacramento Valley, California, USA. Arch. Environ. Contamin. Toxicol. 56:577-587.

Zechmeister H.G., Hagendorfer H., Hohenwallner D., Hanus-Illnar A. \& Riss A. 2006. Analyses of platinum group elements in mosses as indicators of road traffic emissions in Austria. Atmospheric Environment 40:77207732 . 